\title{
Colour image denoising by eigenvector analysis of neighbourhood colour samples
}

\author{
Pedro Latorre Carmona • Juan José Miñana · Samuel Morillas
}

Received: date / Accepted: date

\begin{abstract}
Colour image smoothing is a challenging task because it is necessary to appropriately distinguish between noise and original structures, and to smooth noise conveniently. In addition, this processing must take into account the correlation among the image colour channels. In this paper, we introduce a novel colour image denoising method where each image pixel is processed according to an eigenvector analysis of a data matrix built from the pixel neighbourhood colour values. The aim of this eigenvector analysis is threefold: (i) to manage the local correlation among the colour image channels, (ii) to distinguish between flat and edge/textured regions, and (iii) to determine the amount of needed smoothing. Comparisons with classical and recent methods show that the proposed approach is competitive and able to provide significative improvements.
\end{abstract}

Keywords Colour image filter, Colour image smoothing, Eigenvectors, Gaussian noise, Principal Components, Vector Filter.

\section{Introduction}

Image denoising is a topic which has been extensively studied in the fields of computer vision and digital image processing. The denoising (or filtering) step is essential for almost every computer vision system because noise can significantly affect the visual quality of the images as well as the performance of most image processing tasks. Also, in the last years the use of colour images has gained much attention within the computer vision field and therefore colour image denoising has become an important research topic.

Address(es) of author(s) should be given
Among the different sources of noise in digital imaging, probably the most common one is the so-called thermal noise, which is due to the Charge-Coupled Device (CCD) sensor malfunction. This kind of noise is modelled as additive white Gaussian noise. Therefore, the presence of thermal (or Gaussian) noise can be simulated by adding random values from a zero-mean Gaussian distribution to the original values of each image channel independently, where the standard deviation, s, of the Gaussian distribution characterizes the noise intensity [1]. Many methods for reducing image Gaussian noise from colour images have been proposed in the literature, all of them sharing the following goals [1]-[4]:

1. Flat regions should be as smooth as possible, so that the noise is completely removed.

2. Edges and details should be preserved as much as possible, avoiding blurring and sharpening.

3. Texture should not be lost, which means that texture should not be confused with noise.

4. No colour artifacts should be introduced in the denoising process. That is, no new colours different from the original ones in the image should appear after the denoising process.

The earliest approaches for Gaussian noise smoothing were based on a linear approach. These methods, such as the Arithmetic Mean Filter (AMF) [1], are able to suppress noise, because they take advantage of its zero-mean property, but they blur edges and texture significantly. This fact motivated the development of many nonlinear methods that try to overcome this drawback by detecting image edges and details and smoothing them less than the rest of the image.

Within the nonlinear methods, a wide class of them uses averaging to take advantage of the zero-mean prop- 
erty of the noise. This class includes the well-known Bilateral Filter (BF) [5] and its variants [6]-[10]. Also, the works in $[11,12]$ use an averaging operation which is restricted to the (fuzzy) peer group members for each image pixel. Other methods are developed using fuzzy logic or soft switching methods, such as those in [13][22]. Several methods based on different optimizations of weighted averaging are proposed in [23]-[26]. Another important family of filters are the partition based filters [26]-[28] that classify each pixel to be processed into several signal activity categories which, in turn, are associated to appropriate processing methods. Other filters follow a regularization approach [29]-[38] based on the minimization of appropriate energy functions by means of Partial Differential Equations (PDEs). Wavelet theory has also been used to design image filtering methods [39]-[48]. The combination of collaborative and wavelet filtering is proposed in $[49,50]$, and a method using the wavelet transformation and data regularization is proposed in [51]. Other recent methods make use of a combination of image analysis techniques for image segmentation followed by an appropriate smoothing of each image region [52]-[54]. In addition, other methods based on Principal Component Analysis (PCA) in the image spatial domain [55]-[58] have been studied.

On the other hand, it should be pointed out that in the context of colour images, goal 4 in the above list is specially important. It is well-known that colour artifacts may be introduced if the correlation among the image channels is not taken into account [1]-[3]. This implies that the component-wise application of gray-scale methods is not appropriate for colour image processing. A well established solution is the vector approach, which processes images by treating them as vector fields [1]-[3],[5],[9]-[13]. However, it is also known that the denoising capability of vector methods may be inferior to that of the component-wise approaches, for instance, in the case of the Vector Median Filter (VMF) [59] versus the component-wise median or the robust vector median methods $[60,61]$. This means that the development of alternative methods is also interesting. For instance, the work in [14] proposes a different method to manage inter-channel correlation which is based on analyzing the observed differences between colour component couples, and the method in [32] models the correlation by means of colour-ratio constraints.

In this paper, we introduce a new local technique using weighted pixel averaging to approach the colour image denoising problem based on a well known linear algebra tool: eigenvectors. Local eigenvector analysis allows to process the correlation among the colour channels as well as to determine a set of smoothing weighting coefficients to perform the denoising opera- tion. Each image pixel is processed using a data matrix built from its local neighbourhood colour component values. Eigenvector analysis performed on the data matrix provides the necessary information to transform the original data matrix into a new one composed by a new set of uncorrelated variables that can be now processed in a componentwise fashion. Also, eigenvector analysis provides information on the data variance of the new set of variables that we can use to appropriately smooth each one of the new variables, preserving original data and reducing noise. It should be pointed out that the proposed method is completely different from previous PCA methods, as those in [55]-[58], because our analysis is performed in the signal value domain instead of the image spatial domain.

The rest of the paper is organized as follows: Section 2 gives the details about the proposed method; Section 3 presents the experimental results and a systematic comparison against other state-of-the-art methods, which shows that the proposed technique significantly outperforms other local methods of the same family and it is able to compete with non-local methods; Finally, conclusions are drawn in Section 4.

\section{Colour image denoising based on eigenvector analysis}

\subsection{Local data matrix and eigenvector analysis}

The colour image $\mathbf{F}$, which is represented in the RGB colour space, is processed using a sliding filtering window of size $N \times N$ where $N=2 n+1$ and $n=1,2, \ldots$. The sliding window is centered on each pixel to be processed, denoted by $\mathbf{F}_{0}$, which is defined by the tern $\left(F_{0}^{R}, F_{0}^{G}, F_{0}^{B}\right)$ of its three RGB colour components. The rest of the neighbour pixels in the filtering window are denoted as $\mathbf{F}_{i}, i=1, \ldots, N^{2}-1$.

Using the colour component values of the pixels in the filtering window we build a data matrix $\mathbf{D}$ of size $N^{2} \times 3$ where the columns of the matrix are associated to the colour components which are considered as the variables of the data whereas the rows are associated to the pixels that represent the samples in the data set. The main novelty of the method introduced in this paper is that an analysis of the $\mathbf{D}$ matrix is used to: (i) appropriately process the correlation among the image channels, and (ii) to conveniently smooth the noise in the image while preserving the original structures. We propose to perform an eigenvector analysis based on the information provided by the matrix D. For this, we find the eigenvectors, also called characteristic vectors or principal components, of $\mathbf{D}^{\mathbf{T}} \mathbf{D}$, where $T$ denotes matrix transponse. This procedure is behind well-known 
methods such as Singular Value Decomposition (SVD), Independent Component Analysis (ICA) or Principal Component Analysis (PCA) [62,63].

The method of principal components is based on a key result from matrix linear algebra: since $\mathbf{D}^{\mathbf{T}} \mathbf{D}$ is a symmetric matrix, it may be reduced to a diagonal matrix $\mathbf{L}$ by premultiplying and postmultiplying it by a particular orthonormal matrix $\mathbf{O}$ such that the diagonal elements of $\mathbf{L}$ are called the characteristic roots, latent roots or eigenvalues, and the columns of $\mathbf{O}$ are called the characteristic vectors, eigenvectors or latent vectors of $\mathbf{D}^{\mathbf{T}} \mathbf{D}[62,63]$. That is, a vector $\mathbf{v}$ is an eigenvector of $\mathbf{D}^{\mathbf{T}} \mathbf{D}$ if and only if it satisfies that

$$
\mathbf{D}^{\mathbf{T}} \mathbf{D} \mathbf{v}=\lambda \mathbf{v}
$$

where $\lambda$ is a scalar called the eigenvalue corresponding to $\mathbf{v}$ and, for convenience, $\mathbf{v}$ is taken so that it is unitary. Eigenvalues $\lambda_{i}$ of $\mathbf{D}^{\mathbf{T}} \mathbf{D}$ can be obtained as the solutions of the equation

$$
\operatorname{det}\left(\mathbf{D}^{\mathbf{T}} \mathbf{D}-\lambda \mathbf{I}\right)=\mathbf{0},
$$

where det denotes the matrix determinant. Then, given the non-null eigenvalues $\lambda_{i}$, we can obtain $[62$, 63 ] three associated eigenvectors $\mathbf{v}_{i}$ from the eigenvalue equations

$$
\left(\mathbf{D}^{\mathbf{T}} \mathbf{D}-\lambda_{\mathbf{i}} \mathbf{I}\right) \mathbf{v}_{\mathbf{i}}=\mathbf{0},
$$

that can be considered as an alternative set of orthogonal coordinate axes. Transforming the original data by means of the coordinate axis provided by the eigenvectors implies transforming the original correlated variables into a new set of variables which are uncorrelated. Geometrically, this procedure is simply a principal axis rotation of the original coordinate axis about their means $[62,63]$. Therefore, if we denote by $\mathbf{V}$ the $3 \times 3$ orthonormal matrix that has as columns the three eigenvectors of $\mathbf{D}^{\mathbf{T}} \mathbf{D}$ denoted as $\mathbf{V}^{1}, \mathbf{V}^{2}$, and $\mathbf{V}^{3}$, the mentioned transformation is performed by multiplying $\mathrm{D}$ by $\mathrm{V}$ so that

$$
\mathbf{U}=\mathbf{D V}
$$

where $\mathbf{U}$ denotes the matrix containing the transformed data, also called scores matrix, and each pixel $\mathbf{U}_{i}, i=0, \ldots, N^{2}-1$ is now represented by the tern $\mathbf{U}_{i}=\left(U_{i}^{1}, U_{i}^{2}, U_{i}^{3}\right)$. Moreover, note that, since $\mathbf{V}$ is orthonormal, it is fulfilled that

$\mathbf{U V}^{\mathbf{T}}=\mathbf{D}$
Now, we can directly operate on the values of $\mathbf{U}$ to reduce the noise. Notice that now the columns of $\mathbf{U}$ are associated to a new set of uncorrelated variables that we will denote as $U^{1}, U^{2}$, and $U^{3}$, and which are associated to the eigenvectors $\mathbf{V}^{1}, \mathbf{V}^{2}$, and $\mathbf{V}^{3}$, respectively. This implies that we can safely apply componentwise methods to reduce the noise independently in each of the new variables. In particular, we propose to apply the method described in Section 2.2 which, as it will be explained in the following, is devised to take advantage of the information provided by this eigenvector analysis. Finally, denoised data need to be transformed again into the RGB space. According to Eq. (5), this can be done by simply multiplying the data in the transformed space by the matrix $\mathbf{V}^{\mathbf{T}}$.

It should be stressed that, even though from a strictly theoretical point of view obtaining 3 -component eigenvectors from small datasets does not guarantee useful results for the low ratio of data samples per variable, in our case, we see that the results for small filtering windows $(3 \times 3$, which means 9 data) are satisfactory and useful. This is probably due to the strong correlation among the colour channels and the high spatial redundancy (spatial correlation) shown in digital images. Data could be increased by using a larger filtering window, but it is known that large windows lead to undesired blurring in the image and, consequently, we prefer to use small ones. Note that a number of works already exist where eigenvectors are computed satisfactorily even with a much lower ratio of data samples per variable. For instance, this happens in PCA-based modelling of industrial batch processes [64,65], where thousands of variables are analyzed using less than a hundred samples. So, this practice is quite common, indeed.

\subsection{Denoising method}

To devise an appropriate denoising method using the information from the previous eigenvector analysis we take advantage of the following properties of the eigenvectors: (i) eigenvectors are obtained as orthogonal linear combinations of the original variables; (ii) these linear combinations are obtained so that the sample variance of the original data is maximized $[62,63]$. This means that one of the eigenvectors, also called the first principal component, is obtained so that its direction corresponds to the direction of maximum sample variance in the original variable space; then, another eigenvector, called the second principal component, is obtained as the vector whose direction is orthogonal to the previous one and that maximizes the remaining variance, and so forth. 
In colour images, edges, texture and image details can be seen as correlated variations of the RGB values. On the other hand, since Gaussian noise in the image channels is commonly assumed to be independent, variations due to noise are uncorrelated. Then, because of the explanation above, correlated variations due to edges or image details, can only be associated to one of the eigenvectors $\mathbf{V}^{i}$ since, otherwise, the new variables would not be uncorrelated. An example of this is given in Figure 1 (see supplementary material) where we show an edge in a colour image along with the original colour component values and the result after applying the eigenvector transformation on the data (for a better visualization, only the $\mathrm{R}$ and $\mathrm{G}$ values are used). On the other hand, Figure 2 (see supplementary material) corresponds to a flat region of a colour image where it can be seen that this effect is not observed.

Also, because of the sample variance maximization feature, we can identify the new variable $U^{i}$ associated to the eigenvector that explains the correlated data variation due to an edge or image detail as the variable $U^{i}$ that fulfills that $\sigma\left(U^{i}\right)>>\sigma\left(U^{j}\right)$ and $\sigma\left(U^{i}\right)>>$ $\sigma\left(U^{k}\right)$, where $\sigma$ denotes the sample standard deviation. In such a case, since data represented by $U^{i}$ is associated to image edge or detail information and it is also noisy, this variable should not be excessively smoothed, whereas $U^{j}$ and $U^{k}$ can be safely smoothed since they do not bear image information but mainly noise. On the other hand, in flat image regions where only uncorrelated variations due to noise in the data are observed, it is expected that the variances of $U^{i}, U^{j}$ and $U^{k}$ are similar, that is, $\sigma\left(U^{i}\right) \approx \sigma\left(U^{k}\right) \approx \sigma\left(U^{j}\right)$, which means that the three variables can be safely smoothed. This fact is shown in Figure 3 (see supplementary material) where we give two gray-scale images corresponding to two noisy images where the gray level of each pixel is proportional to the maximum of $\sigma\left(U^{i}\right), \sigma\left(U^{j}\right)$, and $\sigma\left(U^{k}\right)$. We can easily see that brightest image areas correspond to image edges and details. According to this, we devise the denoising method to smooth these brighter areas less than the darker ones.

As mentioned in the Introduction, we aim at applying a weighted averaging operation in order to smooth each component independently. Then, to smooth each component of the pixel represented by the tern $\mathbf{U}_{0}=$ $\left(U_{0}^{1}, U_{0}^{2}, U_{0}^{3}\right)$, the operation given by the following expression is applied:

$$
\hat{U}_{0}^{i}=\frac{\sum_{p=0}^{N^{2}-1} W_{p}^{i} U_{p}^{i}}{\sum_{p=0}^{N^{2}-1} W_{p}^{i}}, i=1,2,3 .
$$

where $i$ refers to the colour channel and $p$ to the pixel number in the neigbourhood window around a pixel.

According to above, the weights $W_{p}^{i}$ should be computed so that the component $U_{0}^{i}$ is less smoothed when $\sigma\left(U^{i}\right)>>\sigma\left(U^{j}\right)$ and $\sigma\left(U^{i}\right)>>\sigma\left(U^{k}\right)$, and more smoothed otherwise. For this, we define the normalized standard deviation $\sigma_{n}$ of a variable $U^{i}$ as

$$
\sigma_{n}\left(U^{i}\right)=\frac{\sigma\left(U^{i}\right)}{\sum_{j=1}^{3} \sigma\left(U^{j}\right)}
$$

To appropriately perform the averaging, the weights $W_{p}^{i}$ should be computed using a decreasing function on $\left|U_{p}^{i}-U_{0}^{i}\right|$ so that only $U_{p}^{i}$ values close to $U_{0}^{i}$ receive high weights. For this, we use the following exponential based expression, but any other decreasing function could be used instead, as well:

$W_{p}^{i}=\exp \left(-\frac{\left|U_{p}^{i}-U_{0}^{i}\right| \sigma_{n}\left(U^{i}\right)}{D}\right)$,

where $D>0$ is a filter parameter that tunes the global smoothing capability of the method. It can be seen that larger values of $D$ imply that values of $W_{p}^{i}$ will be closer to 1 and, therefore, the smoothing capability is higher. Conversely, for lower values of $D$, the smoothing capability decreases. The appropriate setting of $D$ will be experimentally studied in Section 3.1. Note that the value given by $\sigma_{n}\left(U^{i}\right)$ is also related to the smoothing capability: for lower values of $\sigma_{n}\left(U^{i}\right)$ the smoothing capability increases whereas for higher values of $\sigma_{n}\left(U^{i}\right)$ the smoothing performed is lower. Consequently, the desired behaviour is achieved.

Finally, the processing scheme proposed in Sections 2.1 and 2.2 is summarized in Algorithm 1.

\section{Experimental results and comparisons}

In the experimental section we have used the test images Pills, Parrots, Lenna, Beach, Headphones, Flower, and Grass. We have extracted patches (Fig. 4, see supplementary material) of the original images with different (small) sizes and texture and detail content to test the methods against different varieties of spatial frequencies and also to better appreciate the performance differences among different parameter settings and filtering methods. These images have been corrupted with noise using the classical white additive Gaussian model [1]. Each colour image channel has been contaminated independently with a varying standard deviation, $s$, of 


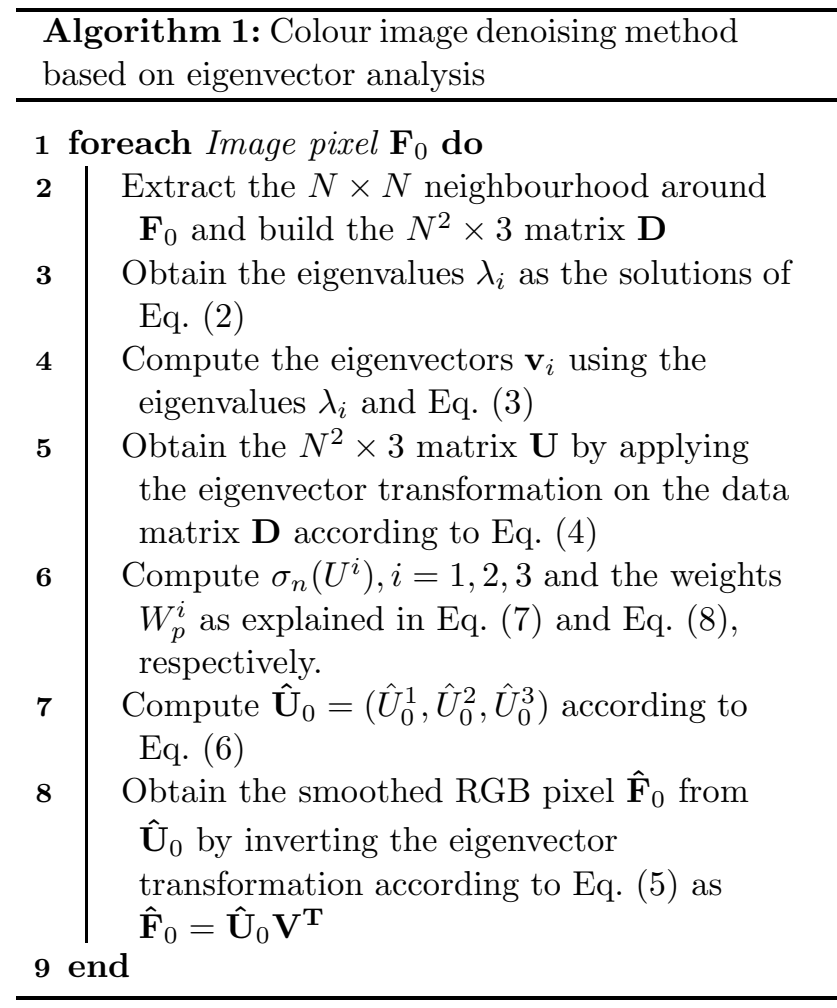

the Gaussian distribution, which represents the noise intensity.

To assess the performance of a filtering process, we use five different measures each of them accounting for a different point of view of assessment: the Mean Absolute Error (MAE) [1] to measure the detail preserving ability; the Peak Signal to Noise Ratio (PSNR) [1] for the noise suppression ability; the Normalized Colour Difference (NCD) [1] for the colorimetric preservation ability; the generalization to colour of the popular structural similarity measure [67] named Fuzzy Colour Structural Similarity (FCSS) [68]; and the perceptual difference inspired in the image colour appearance model iCAM (iCAMd) [69], which we have used setting for all images 5 degrees of visual angle as visualization conditions.

\subsection{Parameter setting}

In order to choose the appropriate adjustment of the filter parameter $D$ in Eq. (8), we have experimentally analyzed the filter performance in terms of PSNR as a function of $D$ using the images Pills and Lenna (Fig. 4 (a),(c), see supplementary material). They have been contaminated with varying standard deviation $s$ of Gaussian noise. For each value of $s \in[1,30]$, we have experimentally determined the optimal setting for $D$ in terms of the PSNR quality measure. The obtained results, which are shown in Fig. 5 (see supplementary material), suggest that for values of the noise standard deviation $s$ in $[1,30]$, an appropriate value of $D$ can be set proportionally to $s$ in the $[1,25]$ interval. So, roughly, $D$ can be set according to the linear relation

$$
D=\frac{5}{6} s,
$$

where the standard deviation of the corrupting Gaussian noise $s$ can be estimated using the method in [66]. Also, in Fig. 6 (see supplementary material), we represent the performance in terms of PSNR as a function of $D$ for four noisy images. It can be seen that it is not necessary to optimally set $D$ in order to achieve a superior performance and, therefore, the proposed automatic setting for $D$ is sufficient.

\subsection{Comparison with state-of-the-art methods}

The performance of the proposed filter is compared against the following filters: Bilateral Filter (BF) [5], Adaptive Nearest Neighbour Filter (ANNF) [13], Chromatic filter [23] (CHRF), Fuzzy Vector Median Filter (FVMF) [15], Peer Group Averaging (PGA) [11], Fuzzy Directional Derivative Filter [18] (FDDF), Fuzzy Noise Reduction Filter [14] (FNRF), Fuzzy Wavelet Denoising method (FWD) [42], Collaborative Wavelet Filter (CWF) $[49,50]$, and Colour Regularization Filter (CRF) [32]. All filters have been applied on a $3 \times 3$ filter window in an iterative fashion with the same stop condition: the method stops when it reaches the maximum performance in terms of PSNR, ignoring the iteration for which PSNR decreases for the first time. For each method, the parameter setting advised by the respective authors has been employed, tuning experimentally when necessary.

Experimental results are presented in Tables 1-7 (see supplementary material). In each table, the best result for each noise level and performance measure is written in red, and the second best, in blue. These Tables show that the proposed method exhibits one of the best overall performance for all quality measures, implying that the proposed method achieves a good noise reduction without introducing colour artifacts, as well as it properly preserves image details, colors and image structures. From these results, it is easy to identify a group of filters, most of them based on averaging pixel values for smoothing, with performance significantly lower than the rest: BF, ANNF, CHRF, FVMF, PGA, and FDDF filters. On the other hand, the fuzzy filters FWD and FNRF, and the CRF show a better performance than the group before. More specifically, FNRF and CRF are able to yield a competitive performance in some cases: FNRF performs very well for im- 
ages with a higher spatial frequency content when they are contaminated with medium to high noise intensities; CRF also performs competitively when the noise intensity is high, specially in terms of structural similarity. However, the best performance is achieved by the non-local method CWF and the proposed method, as they rank first or second in more than $70 \%$ of the cases. Furthermore, we can see that the proposed method is, in overall, better than CWF for images with a higher spatial frequency content (more texture and small details) whereas CWF is better for the larger images that have a lower spatial frequency content, that is, more and larger areas of homogeneous regions. This is logical as the block matching procedure applied in CWF finds more matches in this type of images, which allows to achieve a higher denoising performance. On the other hand, this indicates that our method lacks noise reduction capability in homogeneous regions, which is a point to improve in the future. Finally, it should be stressed that the performance of the proposed method is significantly better than other filters in the local averaging family, and it is able to compete with non-local methods.

Also, some images denoised using the most competitive filters are shown in Figures 7-13 (see supplementary material). By visually inspecting these results, we can see that the images generated by the proposed method are visually pleasing, specially for images with a high spatial frequency content and from the detail and structure preservation point of view. The following points may be stressed:

- Figure 7: All FWD, CWF, CRF and the proposed method perform quite well in this case. It seems that the proposed method preserves better the texture in the image (see right-up area) whereas FWD and CWD reduce better the noise but blur the texture a little.

- Figure 8: In these images we can see that the FWD and CWF methods are generating a little blurry output images. Also, FWD has introduced some colour artifacts (next to the eye). CRF has reduced noise well but it has also introduced a little bit of blur around the eye and in some edges, and the proposed method has better preserved edges and texture (specially around the eye) while appropriately reducing the noise.

- Figure 9: Here, according to the quantitative results, CWF performs the best: It removes the noise and keeps all texture and details in the image. CRF and the proposed method are able to remove the noise but blur texture a bit. FNRF does not blur the texture but it does not remove all the background noise.
- Figure 10: In these images we can see clear performance differences among the FNRF, FWD, CRF and the proposed method. The FNRF does not reduce all noise in the image and some of the image edges are sharpened, so, they are not perfectly preserved. FWD blurs the image too much and, in addition, it introduces some colour artifacts. CRF is able to reduce well the noise but it blurs the edges in the image. Finally, we can see that the proposed method generates a more visually pleasing image where the noise is reduced and the edges are preserved without sharpening them.

- Figure 11: In this example we can see that CRF has sometimes problems to preserve colour information in the image, probably because of a deficient processing of image inter-channel correlation. Also, we see that FNRF and CWF blur the image more than the proposed method, which is the one achieving here the best trade-off between noise reduction and details preservation.

- Figure 12: In this case, the noise reduction capability of CWF stands out: CWF reduces all noise and keeps the main details but blurs a bit smaller details. On the other hand, the noise reduction capability of FNRF is below the rest. CRF and the proposed method perform quite similar, which agrees with the results in terms of FCSS: they are best in keeping the global image structure. While they do not remove the noise as well as CWF, they preserve better hair textures.

- Figure 13: In this example, it can be seen that both FNRF and CRF are not able to properly reduce all noise. FWD reduces the noise but the output image is too blurry and it also contains too many colour artifacts. The proposed method seems also here to produce the best results since it is able to reduce the noise but in this case some edges and texture have been a little blurred.

\section{Conclusions}

In this paper, we have introduced a local method to reduce Gaussian noise from colour images which is based on an eigenvector analysis of the colour samples in each pixel neighbourhood. The proposed method employs a local procedure both to appropriately process the correlation among the colour image channels, as well as to compute a set of weighting coefficients which are used to smooth each pixel in the image. It performs well in colour image denoising since it is able to reduce image noise while preserving image edges, texture and other details without introducing colour artifacts. Experimental results have shown that the pro- 
posed method is able to exhibit a high performance which is competitive with respect to recent state-of-theart methods both from the quantitative as well as from the visual point of view. The proposed method clearly outperforms other local methods and it is competitive with non-local ones.

\section{References}

1. Plataniotis, K.N., Venetsanopoulos, A.N.: Color Image processing and applications. Springer-Verlag, Berlin (2000).

2. Lukac, R., Smolka, B., Martin, K., Plataniotis, K.N., Venetsanopoulos, A.N.: Vector Filtering for Color Imaging, IEEE Signal Processing Magazine, Special Issue on Color Image Processing, 22, 74-86 (2005).

3. Lukac, R., Plataniotis, K.N.: A taxonomy of color image filtering and enhancement solutions, in Hawkes, P.W. (ed) Advances in Imaging and Electron Physics, 140, pp. 187-264. Elsevier Acedemic Press (2006).

4. Buades, A., Coll, B., Morel, J.M.: Nonlocal image and movie denoising, International Journal of Computer Vision, 76, 123-139 (2008).

5. Tomasi, C., Manduchi, R.: Bilateral filter for gray and color images, in Proceedings of IEEE International Conference Computer Vision, 839-846 (1998).

6. Elad, M.: On the origin of bilateral filter and ways to improve it, IEEE Transactions on Image Processing, 11, 1141-1151 (2002).

7. Kao, W.C., Chen, Y.J.: Multistage bilateral noise filtering and edge detection for color image enhancement, IEEE Transactionc on Consumer Electronics, 51, 13461351 (2005).

8. Garnett, R., Huegerich, T., Chui, C., He, W.: A universal noise removal algorithm with an impulse detector, IEEE Transactions on Image Processing, 14, 1747-1754 (2005).

9. Morillas, S., Gregori, V., Sapena, A.: Fuzzy Bilateral Filtering for color images, Lecture Notes in Computer Science, 4141, 138-145 (2006).

10. Zhang, B., Allenbach, J.P.: Adaptive bilateral filter for sharpness enhancement and noise removal, IEEE Transactions on Image Processing, 17, 664-678 (2008).

11. Kenney, C., Deng, Y., Manjunath, B.S., Hewer, G.: Peer group image enhancement, IEEE Transactions on Image Processing, 10, 326-334 (2001).

12. Morillas, S., Gregori, V., Hervás, A.: Fuzzy peer groups for reducing mixed Gaussian-impulse noise from color images, IEEE Transactions on Image Processing, 18, 14521466 (2009).

13. Plataniotis, K.N., Androutsos, D., Venetsanopoulos, A.N.: Adaptive fuzzy systems for multichannel signal processing, The Proceedings of the IEEE, 87, 1601-1622 (1999).

14. Schulte, S., De Witte, V., Kerre, E.E.: A fuzzy noise reduction method for colour images, IEEE Transactions on Image Processing, 16, 1425-1436 (2007).

15. Shen, Y., Barner, K.: Fuzzy vector median-based surface smoothing, IEEE Transactions on Visualization and Computer Graphics, 10, 252-265 (2004).

16. Lukac, R., Plataniotis, K.N., Smolka, B., Venetsanopoulos, A.N.: cDNA Microarray Image Processing Using Fuzzy Vector Filtering Framework, Fuzzy Sets and Systems, 152, 17-35 (2005).
17. Smolka, B.: On the new robust algorithm of noise reduction in color images, Computers \& Graphics, 27, 503-513 (2003).

18. Van de Ville, D., Nachtegael, M., Van der Weken, D., Philips, W., Lemahieu, I., Kerre, E.E.: Noise reduction by fuzzy image filtering, IEEE Transaction on Fuzzy Systems, 11, 429-436 (2003).

19. Schulte, S., De Witte, V., Nachtegael, M., Van der Weken, D., Kerre, E.E.: Histogram-based fuzzy colour filter for image restoration, Image and Vision Computing, 25, 1377-1390 (2007).

20. Nachtegael, M., Schulte, S., Van der Weken, D., De Witte, V., Kerre, E.E.: Gaussian noise reduction in grayscale images, International Journal of Intelligent Systems Technologies and Applications, 1, 211-233 (2006).

21. Schulte, S., De Witte, V., Nachtegael, M., Mélange, T., Kerre, E.E.: A New Fuzzy Additive Noise Reduction Method, Lecture Notes in Computer Science, 4633, 12-23 (2007).

22. Morillas, S., Schulte, S., Mélange, T., Kerre, E.E., Gregori, V.: A soft-switching approach to improve visual quality of colour image smoothing filters, in Proceedings of Advanced Concepts for Intelligent Vision Systems ACIVS07, Lecture Notes in Computer Science, 4678, 254261 (2007).

23. Lucchese, L., Mitra, S.K.: A new class of chromatic filters for color image processing: theory and applications, IEEE Transactions on Image Processing, 13, 534-548 (2004).

24. Lee, J.A., Geets, X., Grégoire, V., Bol, A.: Edgepreserving filtering of images with low photon counts, IEEE Transactions on Pattern Analysis and Machine Intelligence, 30, 1014-1027 (2008).

25. Russo, F.: Technique for image denoising based on adaptive piecewise linear filters and automatic parameter tuning, IEEE Transactions on Instrumentation and Measurement, 55, 1362-1367 (2006).

26. Shao, M., Barner, K.E.: Optimization of partition-based weighted sum filters and their application to image denoising, IEEE Transactions on Image Processing, 15, 1900-1915 (2006).

27. Ma, Z., Wu, H.R., Feng, D.: Partition Based Vector Filtering Technique for Suppression of Noise in Digital Color Images, IEEE Transactions on Image Processing, 15, 2324-2342 (2006).

28. Ma, Z., Wu, H.R., Feng, D.: Fuzzy Vector Partition Filtering Technique for Color Image Restoration, Computer Vision and Image Understanding, 107, 26-37 (2007).

29. Perona, P., Malik, J.: Scale-space and edge detection using anisotropic diffusion, IEEE Transactions on Pattern Analysis and Machine Intelligence, 12, 629-639 (1990).

30. Sroubek, F., Flusser, J.: Multichannel blind iterative image restoration, IEEE Transactions on Image Processing 12, 1094-1106 (2003).

31. Hu, J., Wang, Y., Shen, Y.: Noise reduction and edge detection via kernel anisotropic diffusion, Pattern Recognition Letters, 29, 1496-1503 (2008).

32. Li, X.: On modeling interchannel dependency for color image denoising, International Journal of Imaging Systems and Technology, Special issue on applied color image processing, 17, 163-173 (2007).

33. Keren, D., Gotlib, A.: Denoising Color Images using regularization and correlation terms, Journal of Visual Communication and Image Representation, 9, 352-365 (1998).

34. Lezoray, O., Elmoataz, A., Bougleux, S.: Graph regularization for color image processing, Computer Vision and Image Understanding, 107, 38-55 (2007). 
35. Elmoataz, A., Lezoray, O., Bougleux, S.: Nonlocal discrete regularization on weighted graphs: A framework for image and manifold processing,IEEE Transactions on Image Processing, 17, 1047-1060 (2008).

36. Blomgren, P., Chan, T.: Color TV: total variation methods for restoration of vector-valued images, IEEE Transactions on Image Processing, 7, 304-309 (1998).

37. Tschumperl, D., Deriche, R.: Vector-valued image regularization with PDEs: A Common framework from different applications, IEEE Transactions on Pattern Analysis and Machine Intelligence, 27, 506-517 (2005).

38. Plonka, G., Ma, J.: Nonlinear regularized reactiondiffusion filters for denoising of images with textures, IEEE Transactions on Image Processing, 17, 1283-1294 (2007).

39. Melange, T., Zlokolica, V., Schulte, S., De Witte, V., Nachtegael, M., Pizurca, A., Kerre, E.E., Philips, W.: A new fuzzy motion and detail adaptive video filter, Lecture Notes in Computer Science, 4678, 640-651 (2007).

40. De Backer, S., Pizurica, A., Huysmans, B., Philips, W., Scheunders, P.: Denoising of multicomponent images using wavelet least-squares estimators, Image and Vision Computing, 26, 1038-1051 (2008).

41. Dengwen, Z., Wengang, C.: Image denoising with an optimal threshold and neighboring window, Pattern Recognition Letters, 29, 1694-1697 (2008).

42. Schulte, S., Huysmans, B., Pizurica, A., Kerre, E.E., Philips, W.: A New Fuzzy-Based Wavelet Shrinkage Image Denoising Technique, in Proceedings of Advanced Conceptps for Intelligent Vision Systems ACIVS06, Lecture Notes in Computer Science, 4179, 12-23 (2006).

43. Pizurica, A., Philips, W.: Estimating the probability of the presence of a signal of interest in multiresolution single and multiband image denoising, IEEE Transactions on Image Processing, 15, 654-665 (2006).

44. Scheunders, P.: Wavelet thresholding of multivalued images, IEEE Transactions on Image Processing, 13, 475483 (2004).

45. Sendur, L., Selesnick, I.W.: Bivariate shrinkage functions for wavelet-based denoising exploiting interscale dependency, IEEE Transactions on Signal Processing, 50, 27442756 (2002)

46. Balster, E.J., Zheng, Y.F., Ewing, R.L.: Feature-based wavelet shrinkage algorithm for image denoising, IEEE Transactions on Image Processing, 14, 2024-2039 (2005).

47. Miller, M., Kingsbury, N.: Image denoising using derotated complex wavelet coefficients, IEEE Transactions on Image Processing, 17, 1500-1511 (2008).

48. Zhang, B., Fadili, J.M., Starck, J.L.: Wavelets, Ridgelets, and Curvelets for poisson noise removal, IEEE Transactions on Image Processing, 17, 1093-1108 (2008).

49. Dabov, K., Foi, A., Katkovnik, V., Egiazarian, K.: Image denoising by sparse 3D transform-domain collaborative filtering, IEEE Transactions on Image Processing, 16, 2080-2095 (2007).

50. Dabov, K., Foi, A., Katkovnik, V., Egiazarian, K.: Color image denoising via sparse $3 \mathrm{D}$ collaborative filtering with grouping constraint in luminance-chrominance space, in Proceedings of the IEEE International Conference on Image Processing ICIP2007 , 313-316 (2007).

51. Hao, B.B., Li, M., Feng, X.C.: Wavelet iterative regularization for image restoration with varying scale parameter, Signal Processing: Image Communication, 23, 433-441 (2008).

52. Zhao, W., Pope, A.: Image restoration under significat additive noise, IEEE Signal Processing Letters, 14, 401404 (2007).
53. Gijbels, I., Lambert, A., Qiu, P.: Edge-preserving image denoising and estimation of discontinuous surfaces, IEEE Transactions on Pattern Analysis and Machine Intelligence, 28, 1075-1087 (2006).

54. Liu, C., Szeliski, R., Kang, S.B., Zitnik, C.L.,Freeman, W.T.: Automatic estimation and removal of noise from a single image, IEEE Transactions on Pattern Analysis and Machine Intelligente, 30, 299-314 (2008).

55. Oja, E.: Principal components, minor components, and linear neural networks, Neural Networks, 5, 927-935 (1992).

56. Takahashi, T.: Kurita, T.: Robust de-noising by kernel PCA, in proceedings of ICANN2002, Lecture Notes in Computer Science, 2145, 739-744 (2002).

57. Park, H., Moon, Y.S.: Automatic Denoising of 2D color face images using recursive PCA reconstruction, in Proceedings of Advanced Conceptps for Intelligent Vision Systems ACIVS06, Lecture Notes in Computer Science, 4179, 799-809 (2006).

58. Teixeira, A.R., Tom, A.M., Stadlthanner, K., Lang, E.W.: KPCA denoising and the pre-image problem revisited, Digital Signal Processing, 18, 568-580 (2008).

59. Astola, J., Haavisto, P., Neuvo, Y.: Vector Median Filters, Proceedings of the IEEE, 78, 678-689 (1990).

60. S. Morillas, V. Gregori, A. Sapena, Adaptive marginal median filter for colour images, Sensors 11, 3205-3213 (2011).

61. S. Morillas, V. Gregori, Robustifying Vector Median Filter, Sensors 11, 8115-8126 (2011).

62. Dillon, W.R., Goldstein, M.: Multivariate analysis: Methods and applications. John Wiley \& Sons (1984).

63. Jackson, J.E.: A user's guide to principal components. Wiley, (2003).

64. Camacho, J., Picó, J.: Multi-phase principal component analysis for batch processes modelling, Chemometrics and Intelligent Laboratory Systems, 81, 127-136 (2006).

65. Nomikos, P., MacGregor, J.: Multivariate SPC charts for monitoring batch processes, Technometrics 37, 4159 (1995).

66. Immerkaer, J.: Fast noise variance estimation, Computer Vision and Image Understanding, 64, 300-302 (1996).

67. Z. Wang, A.C. Bovik, H.R. Sheikh, E.P. Simoncelli, Image Quality Assessment: From Error Visibility to Structural Similarity, IEEE Transactions on Image Processing 13(4)(2004)600-612.

68. Svetlana Grecova, Samuel Morillas, Perceptual similarity between color images using fuzzy metrics, Journal of Visual Communication and Image Representation 34 (2016) 230-235.

69. M.D. Fairchild, G.M. Johnson, iCAM framework for image appearance differences and quality, Journal of Electronic Imaging 13 (1) (2004) 126-138 